\title{
Identification at Biovar Level of Brucella Isolates Causing Abortion in Small Ruminants of Iran
}

\author{
Ali Mohammad Behroozikhah, ${ }^{1}$ Ramin Bagheri Nejad, ${ }^{1}$ \\ Karim Amiri, ${ }^{2}$ and Ali Reza Bahonar ${ }^{3}$ \\ ${ }^{1}$ Brucellosis Department, Razi Vaccine and Serum Research Institute, Karaj 3197619751, Iran \\ ${ }^{2}$ Office of Brucellosis Control and Eradication, Iranian Veterinary Organization, Tehran 1595814111, Iran \\ ${ }^{3}$ Food Hygiene and Quality Control Department, Faculty of Veterinary Medicine, University of Tehran, Tehran 1419963111, Iran \\ Correspondence should be addressed to Ali Mohammad Behroozikhah, am.behroozikhah@rvsri.ac.ir
}

Received 29 August 2012; Accepted 31 October 2012

Academic Editor: Hin-Chung Wong

Copyright (C 2012 Ali Mohammad Behroozikhah et al. This is an open access article distributed under the Creative Commons Attribution License, which permits unrestricted use, distribution, and reproduction in any medium, provided the original work is properly cited.

\begin{abstract}
To determine the most prevalent biovar responsible for brucellosis in sheep and goat populations of Iran, a cross-sectional study was carried out over 2 years in six provinces selected based on geography and disease prevalence. Specimens obtained from referred aborted sheep and goat fetuses were cultured on Brucella selective media for microbiological isolation. Brucellae were isolated from 265 fetuses and examined for biovar identification using standard microbiological methods. Results showed that 246 isolates (92.8\%) were B. melitensis biovar 1, 18 isolates (6.8\%) were B. melitensis biovar 2, and, interestingly, one isolate (0.4\%) obtained from Mazandaran province was B. abortus biovar 3. In this study, B. melitensis biovar 3 was isolated in none of the selected provinces, and all isolates from 3 provinces (i.e., Chehar-mahal Bakhtiari, Markazi, and Ilam) were identified only as B. melitensis biovar 1 . In conclusion, we found that $B$. melitensis biovar 1 remains the most prevalent cause of small ruminant brucellosis in various provinces of Iran.
\end{abstract}

\section{Introduction}

Brucellosis is a worldwide bacterial zoonosis posing hazards to the public health and causing economic losses for livestock production industry [1-3]. Animal brucellosis is mainly characterized by reproductive involvement resulting in abortion and infertility [2], whereas human brucellosis is a febrile illness known as undulant fever which can lead to chronic debilitating complications $[3,4]$. The disease results from facultative intracellular bacteria belonging to the genus Brucella which are Gram-negative, nonsporeforming and noncapsulated coccobacilli $[3,5,6]$. Currently, the genus consists of 10 species classified based on their host preferences and phenotypic differences $[2,7]$. Three species are also divided into biovars which are $B$. abortus, $B$. melitensis, and B. suis with 7, 3, and 5 biovars, respectively [3].

In sheep and goats, brucellosis is primarily due to $B$. melitensis which is the most pathogenic species for human beings, responsible for the main proportion of human cases in endemic regions including Mediterranean and Middle East countries $[1,3,8-10]$. Humans catch the disease via direct contact with infected animals, their vaginal discharges, and aborted fetuses; dealing with pure cultures; and consumption of unpasteurized contaminated milk and milk products $[3,6,11]$. The latter is the major way through which B. melitensis is transmitted to people in endemic areas $[1,5]$.

Identification of $B$. melitensis biovars involved in small ruminant brucellosis is a critical component of epidemiological surveys required for designing proper preventive and control strategies [8]. In Iran, due to the existence of other related factors including illegal livestock import from neighboring countries where $B$. melitensis is also prevalent and uncontrolled animal movements, it was considered necessary to study changes in the epidemiologic distribution of different biovars in order to determine introduction of new biovars and evaluate the success of ongoing control measures. Therefore, the present study was performed to 
renew our knowledge about the current prevalence status of B. melitensis biovars causing abortion in sheep and goats in Iran.

\section{Materials and Methods}

2.1. Study Design and Sampling. The present cross-sectional study was carried out from 2007 to 2009. According to Iranian Veterinary Organization classification, all country provinces were divided into 3 categories with low $(<2 \%)$, intermediate $(2-3 \%)$, and high $(>3 \%)$ brucellosis prevalence. This classification is based on a seroepidemiological survey carried out in 2003 using conventional rose bengal, serum agglutination, and 2-mercaptoethanol tests. Two provinces were then selected from each category based on geography and availability of a central laboratory in the Provincial Veterinary General Office with skilled personnel for bacterial isolation. The selected provinces were Kerman and Markazi; Ilam and Khorasan Razavi; Chehar-mahal Bakhtiari and Mazandaran from low, intermediate, and high prevalence categories, respectively. All small ruminant aborted fetuses referred to the central laboratory of The Provincial Veterinary General Office during the study period were cultured for brucella isolation. Brucella isolates were sent to Brucellosis Department of Razi Vaccine and Serum Research Institute to be identified at species and biovar levels.

2.2. Isolation of Brucellae. Specimens obtained from liver, lungs, spleen, and fetal stomach content of referred aborted fetuses were cultured on brucella agar (BD, USA) containing $5 \%(\mathrm{v} / \mathrm{v})$ inactivated horse serum, 2\% (w/v) dextrose, and brucella selective supplement (Oxoid, UK) according to the manufacturer's instruction. Tissue specimens were first decontaminated using ethanol and flame and then homogenized in sterile normal saline by means of a grinder. For each specimen, two sets of 3 agar plates were inoculated and incubated at $37^{\circ} \mathrm{C}$ with one set in the air having $10 \%$ $\mathrm{CO}_{2}$. Inoculated plates were monitored every 2 days to detect any colonial growth, and in the case of no colony observation, they were kept at most for 35 days. Suspected colonies based on their morphological characteristics were subcultured on brucella agar slopes for further evaluation and biovar identification.

2.3. Biovar Identification. Brucella isolates, confirmed by microscopic characterization using Gram stain and results of catalase, oxidase, and urease tests, were examined for biotyping according to the standard methods described by Alton et al. [12]. Briefly, biovars were identified based on agglutination with $\mathrm{A}$ and $\mathrm{M}$ monospecific antisera, $\mathrm{CO}_{2}$ requirement for growth, $\mathrm{H}_{2} \mathrm{~S}$ production, lysis by Tbilisi (Tb) and Berkeley $\left(\mathrm{Bk}_{2}\right)$ phages, and growth on media containing $20 \mu \mathrm{g} / \mathrm{mL}$ basic fuchsin and thionin dyes [12-15].

\section{Results}

In the present survey, a total number of 851 aborted sheep and goat fetuses were cultured microbiologically and 265 brucellae were isolated. Biotyping of these 265 isolates showed that $246(92.8 \%)$ were B. melitensis biovar 1. Eighteen isolates $(6.8 \%)$ were identified as $B$. melitensis biovar 2 ; and interestingly, one isolate $(0.4 \%)$, which was obtained from Mazandaran province, was determined as B. abortus biovar 3. Table 1 illustrates numbers of brucella isolates attained in different provinces and results of their biotyping.

B. melitensis biovar 3 was isolated in none of the six provinces during the study period, and $B$. melitensis biovar 2 was only isolated in two provinces which are Khorasan Razavi and Kerman belonging to intermediate and low prevalence categories, respectively.

\section{Discussion}

Small ruminant brucellosis is still a major animal and public health burden in many parts of the world particularly in the Middle East and Mediterranean Region [1, 6, 8, 10]. Sheep and goats are primarily infected by $B$. melitensis as its preferential hosts $[8,18]$. Regarding the public health, $B$. melitensis is the most important zoonotic pathogen amongst the Brucella spp. $[1,3,5]$, accounting for the vast majority of human cases all around the world [8]. In endemic areas, it is transmitted to people mostly through consumption of unpasteurized milk and milk products from sheep and goats $[3,5,19]$.

B. melitensis is divided into three biovars which are differentiated using conventional laboratory methods such as agglutination with $\mathrm{A}$ and $\mathrm{M}$ monospecific antisera and lysis by brucella phages $[12,13]$. Determination of biovars involved in animal brucellosis is an important step for epidemiologic characterization of the disease in any country and a preliminary requirement for designing control and eradication programs [8]. Additionally, owing to the ability of brucellae to adapt to new environments and its reemergence $[3,5,6]$, revealing changes in epidemiologic features of Brucella species/biovars can help to unravel complexity of interactions between the organism, animals, and humans [5].

In Iran, the first B. melitensis isolation from an aborted sheep fetus was reported in $1950[16,17]$. Thereafter it has been widely isolated in different parts of the country mainly from sheep and goats but also occasionally from cattle, camel, and sheepdogs $[17,20]$. All biovars of B. melitensis exist in Iran among which biovar 1 is known as the most widespread. Table 2 summarizes biotyping results of Iranian B. melitensis isolates during two periods of time from 1971 to 2000 .

Immunization of host species with $B$. melitensis strain Rev.1 has been the main strategy for the control of small ruminant brucellosis in Iran since 1960s. During 1983-2003, a test-and-slaughter campaign was also conducted in adult sheep and goats using rose bengal, serum agglutination, and 2-mercaptoethanol tests, while vaccination was limited to young animals. From 2003, control program has been based on mass vaccination using full doses (containing $1-3 \times 10^{9}$ bacteria per dose) of Rev.1 vaccine in lambs and kids at 4-7 months of age, and its reduced doses (containing 0.5-2 
TABLE 1: Number of isolates in different provinces.

\begin{tabular}{|c|c|c|c|c|c|}
\hline \multirow[b]{2}{*}{ Province } & \multirow[b]{2}{*}{ Prevalence category } & \multirow[b]{2}{*}{ Number of isolates } & \multicolumn{3}{|c|}{ Number of different species/biovars } \\
\hline & & & $\begin{array}{c}\text { B. melitensis } \\
\text { biovar } 1\end{array}$ & $\begin{array}{c}\text { B. melitensis } \\
\text { biovar } 2\end{array}$ & $\begin{array}{c}\text { B. abortus } \\
\text { biovar } 3\end{array}$ \\
\hline Mazandaran & High & 55 & 54 & - & 1 \\
\hline Chehar-mahal Bakhtiari & High & 58 & 58 & - & - \\
\hline Khorasan Razavi & Intermediate & 68 & 55 & 13 & - \\
\hline Ilam & Intermediate & 10 & 10 & - & - \\
\hline Markazi & Low & 39 & 39 & - & - \\
\hline Kerman & Low & 35 & 30 & 5 & 一 \\
\hline Total (\%) & & $265(100)$ & $246(92.8)$ & $18(6.8)$ & $1(0.4)$ \\
\hline
\end{tabular}

Table 2: Frequency of different B. melitensis biovars isolated in Iran.

\begin{tabular}{lccccc}
\hline Years & $\begin{array}{c}\text { B. melitensis } \\
\text { biovar 1 }\end{array}$ & $\begin{array}{c}\text { B. melitensis } \\
\text { biovar 2 }\end{array}$ & $\begin{array}{c}\text { B. melitensis } \\
\text { biovar 3 }\end{array}$ & Total & References \\
\hline $1971-1980$ & 851 & 242 & 4 & 1107 & {$[16]$} \\
$1981-2000$ & 2102 & 205 & 106 & 2413 & {$[17]$} \\
\hline Total & 2953 & 447 & 110 & 3510 \\
\hline
\end{tabular}

$\times 10^{6}$ bacteria per dose) in adult female animals. In addition to the mass vaccination, other measures including public education, promotion of sanitary husbandry practices, and microbiological evaluation of abortion outbreaks have been implemented. As a result, the number of new human cases reported annually dropped from 39 in 2005-2006 to 15.9 in 2010-2011 per one hundred thousand people in the country (unpublished data).

Our survey showed that B. melitensis biovar 1 is the most frequent cause of clinical brucellosis in small ruminant populations of the provinces included. This finding is in agreement with the results of the previous studies in the country $[16,17]$. Isolation of $B$. abortus biovar 3 , which is an enzootic etiologic agent of bovine brucellosis [17, 21, 22], from an aborted sheep fetus in our study suggests the possibility of cross-species transmission of the pathogen from cattle. B. abortus has been sporadically identified and reported as a causative agent for sheep brucellosis in Iran $[17,21]$. This indicates that in endemic areas, where both Brucella spp. are present, and cattle and small ruminants are raised in close contact, transmission to nonpreferred hosts may occur $[17,21,23]$. This should be taken into account while implementing control measures.

Whereas the estimation of disease prevalence was not the aim of this study, the results showed that in spite of implementing vaccination and other control measures for years, small ruminant brucellosis in clinical form still persists in various parts of the country. Nevertheless, there is a need for country-wide investigations covering the whole target population including traditionally and nomadically reared flocks [6] to ascertain geographical distribution of different biovars region by region. It will also help trace potential outbreaks, especially in provinces neighboring western and eastern borders. For this purpose, using geographic information system (GIS) will be helpful in analyzing interactions between animal and human brucellosis [24].

\section{Conclusion}

The present study revealed that $B$. melitensis biovar 1 remains the most prevalent cause of clinical form of small ruminant brucellosis in various provinces of Iran. However, more studies are required to determine current status of the disease throughout the country and the evaluation of its impact on human health. Further control and prevention measures can be implemented to curtail the human and animal disease incidence to a greater extent.

\section{Acknowledgments}

The authors would like to thank Dr. F. Esmaeili, S. Ghaem Maghami, M. Seyedin Ghannad, M. Rasht Bafi, M. Azadi, M. H. Moghaddasi, F. Sabbagh Kermani, M. Asadi, A. Ganji, and all personnel of Brucellosis Department at Razi Vaccine and Serum Research Institute for technical assistance.

\section{References}

[1] M. J. Corbel, "Brucellosis: an overview," Emerging Infectious Diseases, vol. 3, no. 2, pp. 213-221, 1997.

[2] P. Nicoletti, "Brucellosis: past, present and future," Prilozi, vol. 31, no. 1, pp. 21-32, 2010.

[3] M. N. Seleem, S. M. Boyle, and N. Sriranganathan, "Brucellosis: a re-emerging zoonosis," Veterinary Microbiology, vol. 140, no. 3-4, pp. 392-398, 2010.

[4] M. P. Franco, M. Mulder, R. H. Gilman, and H. L. Smits, "Human brucellosis," Lancet Infectious Diseases, vol. 7, no. 12, pp. 775-786, 2007.

[5] J. Godfroid, A. Cloeckaert, J. P. Liautard et al., "From the discovery of the Malta fever's agent to the discovery of a marine mammal reservoir, brucellosis has continuously been a re-emerging zoonosis," Veterinary Research, vol. 36, no. 3, pp. 313-326, 2005. 
[6] M. Gwida, S. Al Dahouk, F. Melzer, U. Rösler, H. Neubauer, and H. Tomaso, "Brucellosis-regionally emerging zoonotic disease?" Croatian Medical Journal, vol. 51, no. 4, pp. 289-295, 2010.

[7] P. Wattiau, A. M. Whatmore, M. V. Hessche, J. Godfroid, and D. Fretin, "Nucleotide polymorphism-based single-tube test for robust molecular identification of all currently described Brucella species," Applied and Environmental Microbiology, vol. 77, no. 18, pp. 6674-6679, 2011.

[8] J. M. Blasco, "Control and eradication strategies for brucella melitensis infection in sheep and goats," Prilozi, vol. 31, no. 1, pp. 145-165, 2010.

[9] J. Godfroid and A. Käsbohrer, "Brucellosis in the European Union and Norway at the turn of the twenty-first century," Veterinary Microbiology, vol. 90, no. 1-4, pp. 135-145, 2002.

[10] M. Refai, "Incidence and control of brucellosis in the Near East region," Veterinary Microbiology, vol. 90, no. 1-4, pp. 81-110, 2002.

[11] V. Taleski, L. Zerva, T. Kantardjiev et al., "An overview of the epidemiology and epizootology of brucellosis in selected countries of Central and Southeast Europe," Veterinary Microbiology, vol. 90, no. 1-4, pp. 147-155, 2002.

[12] G. G. Alton, L. M. Jones, R. D. Angus, and J. M. Verger, Techniques for the Brucellosis Laboratory, Institut National de la Recherche Agronomique, Paris, France, 1988.

[13] World Organization for Animal Health (OIE), Manual of Diagnostic Tests and Vaccines for Terrestrial Animals, World Organization for Animal Health, Paris, France, 2009.

[14] M. J. Corbel, Brucellosis in Humans and Animals, World Health Organization, Geneva, Switzerland, 2006.

[15] World Health Organization (WHO), "Joint FAO/WHO expert committee on brucellosis," Sixth Report, Technical Report Series 740, World Health Organization, Geneva, Switzerland, 1986.

[16] E. Zowghi and A. Ebadi, "Typing of brucella strains isolated in Iran," Archives of Razi Institute, vol. 33, pp. 109-114, 1982.

[17] E. Zowghi, A. Ebadi, and M. Yarahmadi, "Isolation and identification of brucella organisms in Iran," Iranian Journal of Clinical Infectious Diseases, vol. 3, no. 4, pp. 185-188, 2008.

[18] B. Garin-Bastuji, J. M. Blasco, M. Grayon, and J. M. Verger, "Brucella melitensis infection in sheep: present and future," Veterinary Research, vol. 29, no. 3-4, pp. 255-274, 1998.

[19] M. Banai, "Control of small ruminant brucellosis by use of Brucella melitensis Rev.1 vaccine: laboratory aspects and field observations," Veterinary Microbiology, vol. 90, no. 1-4, pp. 497-519, 2002.

[20] E. Zowghi and A. Ebadi, "Brucellosis in camels in Iran," Revue Scientifique et Technique, vol. 7, no. 2, pp. 383-386, 1988.

[21] E. Zowghi and A. Ebadi, "Abortion due to brucella abortus in sheep in Iran," Revue Scientifique et Technique, vol. 7, no. 2, pp. 379-382, 1988.

[22] E. Zowghi, A. Ebadi, and B. Mohseni, "Isolation of Brucella organisms from the milk of seronegative cows," Revue Scientifique et Technique, vol. 9, no. 4, pp. 1175-1178, 1990.

[23] E. Zowghi and A. Ebadi, "Naturally occurring brucella melitensis infection in cattle in Iran," Revue Scientifique et Technique, vol. 4, no. 4, pp. 811-814, 1985.

[24] A. A. Haghdoost, L. Kawaguchi, A. Mirzazadeh et al., "Using GIS in explaining spatial distribution of brucellosis in an endemic district in Iran," Iranian Journal of Public Health, vol. 36, no. 1, pp. 27-34, 2007. 


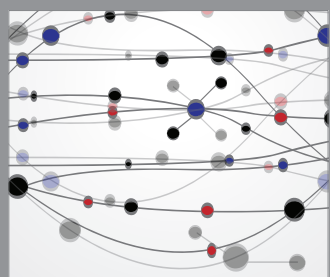

The Scientific World Journal
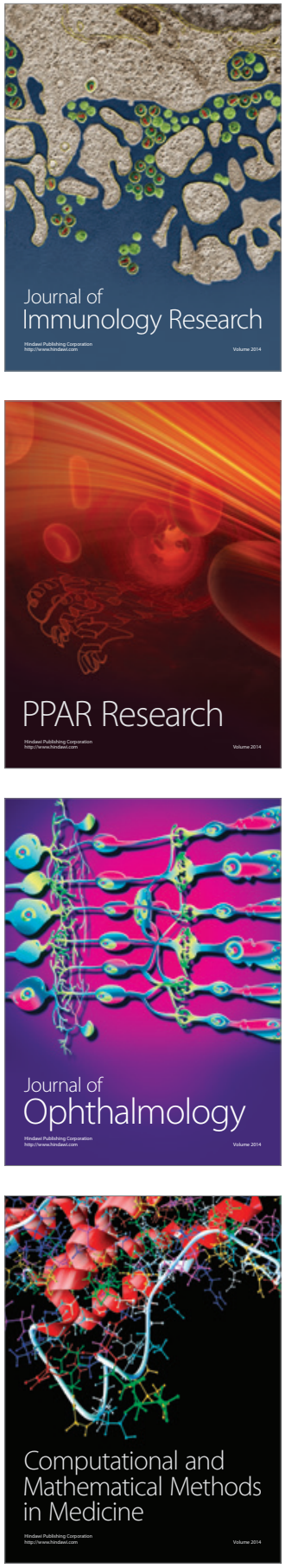

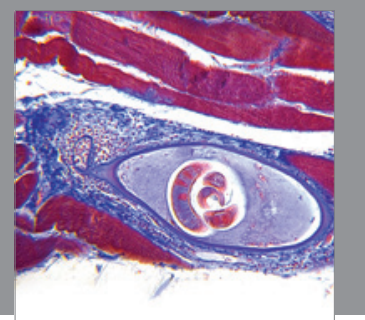

Gastroenterology

Research and Practice
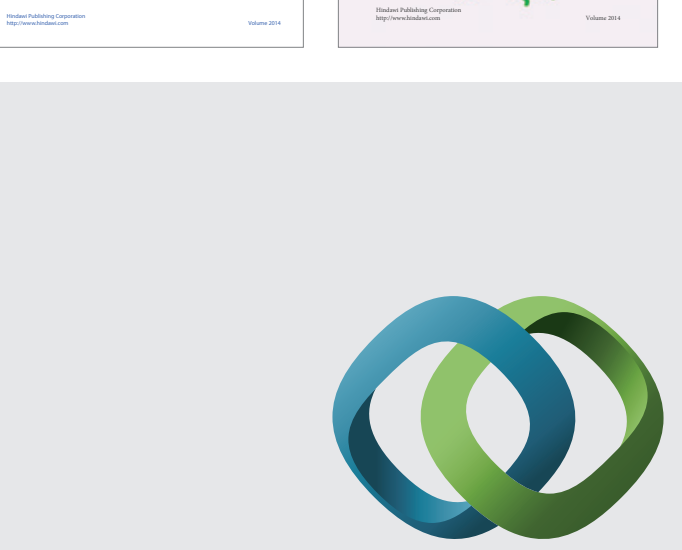

\section{Hindawi}

Submit your manuscripts at

http://www.hindawi.com
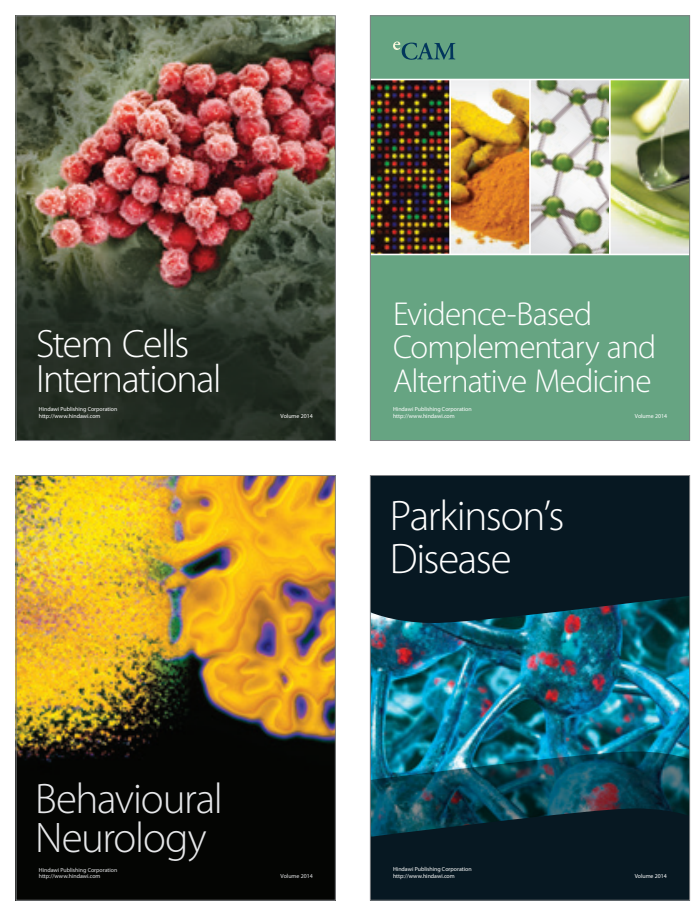

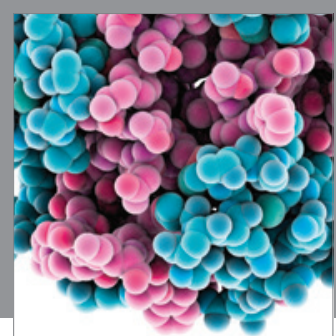

Journal of
Diabetes Research

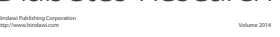

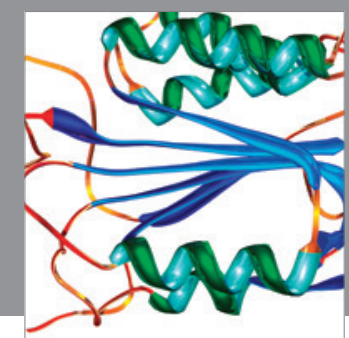

Disease Markers
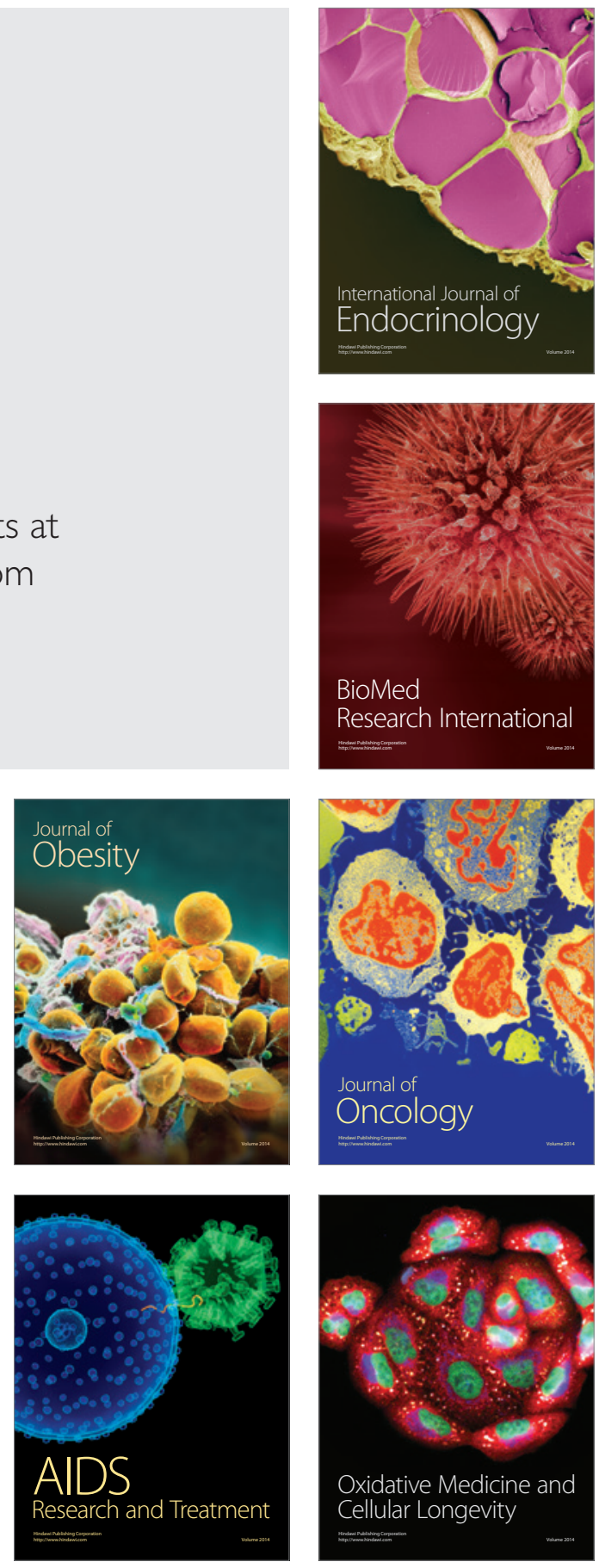